\title{
Analysis of the anatomic subsites, gender and age in unresectable advanced colorectal carcinomas in Tochigi, Japan suggests a shift in location towards the right side colon in elderly patients treated with cetuximab
}

\author{
TOSHIO TANAKA $^{1 *}$, KAZUHITO ICHIKAWA $^{1^{*}}$, TAKASHI MARUOKA $^{1^{*}}$, SHIGEKI TOMITA $^{1 *}$, HIROFUMI UEDA $^{1}$, \\ TAKESHI YAMAGUCHI ${ }^{1,2}$, YOSUKE SHIDA ${ }^{2}$, HIROYUKI KATO ${ }^{2}$, HITOSHI NAGATA ${ }^{3}$, KEIICHI KUBOTA ${ }^{3}$, \\ NAOHIKO AKIMOTO ${ }^{4}$, CHOUITSU SAKAMOTO $^{4}$, JOHJI IMURA $^{5}$, MUNEFUMI ARITA $^{6}$, HIROYUKI TANAKA ${ }^{6}$, \\ YOSUKE OKAMOTO $^{1,7}$, YOSHINORI IGARASHI ${ }^{7}$ and TAKAHIRO FUJIMORI ${ }^{1}$ \\ ${ }^{1}$ Department of Surgical and Molecular Pathology, ${ }^{2}$ First Department of Surgery and ${ }^{3}$ Second Department of Surgery, \\ Dokkyo Medical University School of Medicine, Shimotsuga-Gun, Tochigi 321-0293; ${ }^{4}$ Department of Gastroenterology, \\ Nippon Medical School, Graduate School of Medicine, Bunkyo-ku, Tokyo 113-8603; ${ }^{5}$ Department of Diagnostic Pathology, \\ Graduate School of Medicine and Pharmaceutical Sciences, University of Toyama, Toyama, Toyama 930-0194; \\ ${ }^{6}$ Department of Surgery, Jichi Medical University School of Medicine, Shimotsuke, Tochigi 329-0498; \\ ${ }^{7}$ Division of Gastroenterology and Hepatology, Department of Internal Medicine, Toho \\ University, Omori Medical Center, Ota-ku, Tokyo 143-8541, Japan
}

Received July 30, 2012; Accepted December 12, 2012

DOI: $10.3892 / \mathrm{mco} .2013 .62$

\begin{abstract}
Colorectal cancer is a frequently occurring cancer whose incidence has shown a marked increase in recent years. Additionally, an increase in right side colon in elderly patients has been identified. Therefore, a clinicopathological study was conducted in 49 patients with unresectable advanced colorectal carcinomas to elucidate the association of clinicopathological characteristics and $K$-ras mutation. Of the 49 patients included in this study, 24 were aged $<60$ years with a male/female $(\mathrm{M} / \mathrm{F})$ ratio of $16 / 8$ and 25 patients were aged $\geq 60$ years with a $\mathrm{M} / \mathrm{F}$ ratio of $16 / 9$. Of the patients aged $\geq 65$ years, 15 patients were enrolled as controls and the $M / F$ ratio was $9 / 6$. Results revealed that with regard to the subsite of cancer, unresectable advanced colorectal carcinomas developed in the right-sided colon in 13 patients, left-sided colon in 19 patients and rectum in 17 patients. Right-sided
\end{abstract}

Correspondence to: Professor Takahiro Fujimori, Department of Surgical and Molecular Pathology, Dokkyo Medical University School of Medicine, 880, Kitakobayashi, Mibu, Shimotsuga-Gun, Tochigi 321-0293, Japan

E-mail: hum.pathol@dokkyomed.ac.jp

*Contributed equally

Key words: unresectable advanced colorectal carcinomas, refractory to anti-epidermal growth factor receptor, cetuximab, epidermal growth factor receptor downstream pathway, $K$-ras gene, $b$-raf gene, right side colon colon carcinomas were commonly identified in the elderly patients aged $\geq 65$ years, with a marked tendency in the female patients $(\mathrm{P}=0.024)$. Immunostaining was performed for the epidermal growth factor receptor (EGFR) antibody in 40 patients to determine whether the $K$-ras gene would yield positive results. The mutant $K$-ras gene was identified in 8 patients $(20 \%)$ and the frequency was lower compared with that of the normal colorectal carcinomas. Anti-EGFR antibody (cetuximab) is considered to be a molecularly targeted agent for unresectable advanced colorectal carcinomas. The increase in incidence of right-sided colon carcinomas as well as the increase in the number of patients presenting with colorectal carcinomas means this issue should be addressed. Sessile serrated adenoma/polyp (SSA/P) with $b$-raf mutation and CIMP (CpG island methylator phenotype) abnormality as a precursor lesion of right-sided colon carcinoma is common and since cetuximab refractory wild-type $K$-ras/mutant $b$-raf colorectal carcinoma may increase in elderly patients and patients with right-sided colon carcinoma, a simultaneous examination for the $K$-ras and $b$-raf gene abnormalities for the treatment of colorectal cancer using anti-EGFR antibody (cetuximab) is crucial. In addition, the multidisciplinary assessments regarding the effect of such treatments is likely to be determined based on cumulative results, such as the duration of patient survival.

\section{Introduction}

Although polypectomy has long been recommended for the early detection of and rapid cure from colorectal carcinoma, it has not led to a decrease in colorectal carcinoma (1). 
Colorectal carcinoma is one of the most common cancers worldwide and has the highest incidence of cancer patients by site of the disease in Japan. Additionally, colorectal carcinoma is the second most common cancer after gastric cancer among males as well as the second most common cancer after breast cancer in females, with a significant increase in recent years $(2,3)$. Furthermore, a review of surgical resection cases in elderly patients in the last 10 years has shown a tendency for advanced cancer in the right side colon (4-6). A molecular agent (cetuximab) targeting epidermal growth factor receptor (EGFR) in unresectable advanced colorectal carcinomas (refractory colorectal carcinomas defined by unresectable advanced or metastatic/recurrent colorectal carcinomas) has been developed and is considered to be effective in patients with the wild-type $K$-ras gene (7). $K$-ras gene protein (KRAS), a small $\mathrm{G}$ protein that functions as a signal transducer and downstream integrator of EGFR, is a key component in the EGFR signal cascade. Moreover, $b$-raf gene protein (BRAF) is an immediate downstream effector of $K$-ras in this signaling pathway. However, considering the EGFR signal transduction mechanism and carcinogenic mechanism of colorectal carcinoma, the wild-type $K$-ras/mutant $b$-raf genes are also present $(8,9)$. Carcinomas with these genetic characteristics are described as a serrated neoplasia pathway, which is considered to be characteristic of right-sided colon in elderly patients. Therefore, clarification of clinicopathological features of unresectable advanced colorectal carcinomas may be a clinically important issue when considering the therapeutic modality.

In the present study, 49 cases of unresectable advanced colorectal carcinomas were analyzed to clarify the association between their clinicopathological characteristics and the molecular alterations in $K$-ras gene.

\section{Materials and methods}

Patients and tissue samples. We obtained 49 samples of advanced colorectal carcinomas by surgical resection from 49 patients at the Dokkyo Medical University School of Medicine Hospital (Tochigi, Japan) between January, 2005 and December, 2009. This study was performed following approval by the ethics committee of the Dokkyo Medical University Surgical Pathology. Informed consent was obtained from the patients.

For the ethics procedure, a linkable anonymizing method was used to ensure the study was conducted in a blinded manner. Samples used in this study were materials for biopsy or surgery obtained for diagnosis or treatment and not for research purposes. Participation in the present study did not increase medical disadvantage or risk for patients and data were used strictly for analysis of information as part of therapeutic intervention.

Age and gender of patients, as well as subsites of cancer, were confirmed from the pathological report. The cases were staged using Turnbull's modification of Dukes' classification. Classification of macroscopic type and depth of tumor invasion were assessed according to the Japanese classification of colorectal carcinoma edited by the Japanese Society for Cancer of the Colon and Rectum (JSCCR) (10) and International Union Against Cancer (TNM classification of malignant tumours) (11). Gross classification was as follows: type 1, protuberant
Table I. Patient characteristics (age, gender, gross and microscopic findings) in the unresectable advanced colorectal carcinomas.

\begin{tabular}{lcc}
\hline Patient characteristics & \multicolumn{2}{c}{ No. of patients $(\mathrm{n}=49)$} \\
\hline Age (years) & $<65(\mathrm{n}=34)$ & $\geq 65(\mathrm{n}=15)$ \\
Gender (M:F) & $23: 11$ & $9: 6$ \\
Gross (type) & & \\
1 & $1: 0$ & 0 \\
2 & $13: 6$ & $6: 4$ \\
3 & $7: 5$ & $3: 2$ \\
4 & $1: 0$ & 0 \\
5 & $1: 0$ & 0 \\
Microscopic findings & & \\
Tub 1 & $5: 2$ & $2: 1$ \\
Tub 2 & $12: 8$ & $5: 5$ \\
Por+sig & $5: 1$ & $1: 0$ \\
Others (Muc) & $1: 0$ & $1: 0$ \\
\hline
\end{tabular}

$\mathrm{M} / \mathrm{F}$, male/female; tub 1, well-differentiated adenocarcinoma; tub 2, moderately differentiated adenocarcinoma; por+sig, poorly differentiated adenocarcinoma and signet-ring cell carcinoma; muc, mucinous adenocarcinoma.

type; type 2, ulcerated type with clear margin; type 3, ulcerated type with infiltration; type 4, diffusely infiltrating type and type 5 , unclassified type.

In classifying the carcinoma, the predominant pattern was adopted as its representative histological type. For example, for a tumor comprising mainly of well-differentiated adenocarcinoma with partially moderately differentiated adenocarcinoma or mucinous adenocarcinoma, a diagnosis of well-differentiated type was made. The clinicopathological characteristics of patients are shown in Tables I and II.

\section{Screening of K-ras gene mutations}

DNA extraction. Formalin-fixed, paraffin-embedded samples were cut serially at a thickness of $10 \mu \mathrm{m}$. Based on histolopathological findings, the tumor and corresponding normal tissues were microdissected from each of five serial sections and the tissues were deparaffinized in xylene and rehydrated in graded ethanol series.

DNA was then extracted from the whole dissected tissue using a DNA isolator PS kit (Wako Pure Chemical, Osaka, Japan) according to the supplied protocol.

Analysis of K-ras codon 12 mutations. Extracted DNA was amplified using the polymerase chain reaction (PCR), which was carried out using the following amplification profile: denaturation for $20 \mathrm{~min}$ at $4^{\circ} \mathrm{C}$ once; annealing for $30 \mathrm{sec}$ at $94^{\circ} \mathrm{C}, 30 \mathrm{sec}$ at $55^{\circ} \mathrm{C}$, extension for $30 \mathrm{sec}$ at $72^{\circ} \mathrm{C}$ for 40 cycles; and a final extension for $4 \mathrm{~min}$ at $72^{\circ} \mathrm{C}$. The reaction mixture (100 $\mu \mathrm{l})$ contained $1 \mu \mathrm{g}$ genomic DNA, $10 \mu \mathrm{l}$ Ex Taq buffer, $8 \mu 1 \mathrm{dNTP}$ mixture, 2.5 units Ex Taq polymerase (Takara Bio, Kyoto, Japan), 25 pmol of forward primer (5'-GACT-GAA TAT AAA CTT GTG G-3'), and 25 pmol of reverse primer (3'-CCA GGT CCT GGT AAG AAACT-5'). The DNA 
Table II. Patients characteristics (primary subsites, VI, DM and Dukes' classification) in the unresectable advanced colorectal carcinomas.

\begin{tabular}{|c|c|c|c|c|c|c|c|c|}
\hline \multirow[b]{2}{*}{ Primary tumor site } & \multicolumn{2}{|c|}{ VI } & \multicolumn{2}{|c|}{ DM } & \multicolumn{4}{|c|}{ Dukes' } \\
\hline & $(+)$ & $(-)$ & Liver & Others & A & B & C & D \\
\hline Right side colon & 9 & 0 & 4 & 0 & 0 & 2 & 3 & 4 \\
\hline Left side colon & 23 & 0 & 6 & 3 & 0 & 6 & 9 & 8 \\
\hline Rectum & 16 & 0 & 6 & 0 & 1 & 0 & 10 & 6 \\
\hline
\end{tabular}

VI, vessels invasion; DM, distant metastasis; right-sided colon: cecum, ascending colon and proximal transverse colon; left-sided colon: distal-transverse colon, descending colon and sigmoid colon.

extracted from the gallbladder carcinoma cell line NOZ (kindly provided by Dr S. Nagamori, Jikei University School of Medicine, Tokyo, Japan), in which $K$-ras codon 12 is mutated (12), was used the positive control, whereas DNA from the HeLa cell line, the $K$-ras codon 12 of which is not mutated (13), was used as the negative control.

Mutations of $K$-ras codon 12 in amplified DNA were screened using PCR-restriction fragment length polymorphism (PCR-RFRP) (14) and analysed further by fluorescence direct sequencing. Briefly, the PCR products were digested with $M v a \mathrm{I}$ (Takara, Kyoto, Japan) to distinguish the mutant allele from the wild-type allele and electrophoresed on 3\% agarose gels, followed by staining with ethidium bromide. PCR products encoding the wild-type and mutant alleles were detected as 114 and $143 \mathrm{bp}$ fragments, respectively. Subsequently, the PCR products encoding the mutant allele were extracted from agarose gels using a Sephagras ${ }^{\mathrm{TM}}$ BandPrep kit (Amersham Pharmacia Biotech, Tokyo, Japan) and purified using MicroSpin ${ }^{\mathrm{TM}}$ columns (Amersham Pharmacia Biotech). Extracted DNA was sequenced using a Thermo Sequence ${ }^{\mathrm{TM}}$ fluorescence-labeled primer cycle sequencing kit (Amersham International PLC, Buckinghamshire, UK). The sequence reaction was carried out using the above-mentioned forward primer labeled by the Fluoro-Prime method at its 5 ' end with a fluorescent derivative. The reaction products were analysed using ALF express ${ }^{\mathrm{TM}}$ automatic DNA sequencer (Amersham Pharmacia Biotech).

Immunohistochemical analysis of EGFR protein. Immunohistochemical staining for EGFR was performed with an anti-mouse monoclonal antibody (clone 31G7, Nichirei Biosciences, Inc., Tokyo, Japan) on paraffin-embedded tissue sections using EnVision ${ }^{\mathrm{TM}}+$ Mouse/HRP kit (Dako Japan, Kyoto, Japan). For EGFR, positive and negative controls were used for each set of experiments. Sections of colorectal adenocarcinomas that were confirmed to overexpress this protein were used as positive controls. However, the surrounding normal epithelial cells also showed faint positive cytoplasm staining. Immunoreactivity was considered positive if strong staining of EGFR was detected in at least a focal cytoplasmic membrane (Fig. 1).

Statistical analysis. Data were presented as the means \pm SD. To determine the association of clinicopathological variables
Table III. Differences between subsites of unresectable advanced colorectal carcinomas and age/gender. ${ }^{\mathrm{a}}$

\begin{tabular}{llcl}
\hline & \multicolumn{2}{c}{ No. of patients } & \\
\cline { 2 - 3 } Variables & Age $\geq 65$ years & Age $<65$ years & P-value \\
\hline Female & 4 & & \\
Right side colon & 2 & 1 & 0.024 \\
Left side colon & 0 & 4 & \\
Rectum & & 6 & \\
Male & 3 & 5 & 0.88 \\
Right side colon & 3 & 10 & \\
Left side colon & 3 & 8 & \\
Rectum & & & \\
Total & 7 & 6 & \\
Right side colon & 5 & 14 & \\
Left side colon & 3 & 14 & \\
Rectum & & 5 & \\
\hline
\end{tabular}

${ }^{a}$ For statistical analysis of the association of tumor location with gender and age, Fisher's exact test was used. Statistical analysis was performed using $\mathrm{R}$ (version 2.15.0). $\mathrm{P}<0.05$ was considered to indicate a statistically significant difference.

and tumor location with gender and age, Fisher's exact test was used. Statistical analysis was performed using R (version 2.15.0). $\mathrm{P}<0.05$ was considered to indicate a statistically significant difference.

\section{Results}

Clinical and histopathological features of unresectable advanced colorectal carcinomas. Thirty-two males and 17 females with a mean age of 59.1 years (range, 34-75) were included in this study. All patients had unresectable advanced colorectal carcinomas. There were 34 patients aged $<65$ years with an M/F ratio of 23/11 (female ratio, 32.4\%) and 15 patients were aged $\geq 65$ years with an $\mathrm{M} / \mathrm{F}$ ratio of $9 / 6(40 \%)$. Gross and histological findings were not characterized by the specificity of unresectable colorectal carcinomas. Concerning the subsite of cancer, unresectable advanced colorectal carcinomas developed in right-sided colon in 13 patients, left-sided colon in 19 patients 
Table IV. Primary subsites and status of $K$-ras gene in the unresectable advanced colorectal carcinomas.

\begin{tabular}{llcccccr}
\hline & \multicolumn{3}{c}{ K-ras mutant/<65 } & & \multicolumn{3}{c}{ K-ras mutant $/ \geq 65$} \\
\cline { 2 - 3 } Primary tumor site & Male & Female & Total & & Male & Female & Total \\
\hline Right side colon & $2 / 5$ & $1 / 1$ & $3 / 6$ & & $0 / 3$ & $1 / 4$ & $1 / 7$ \\
Left side colon & $1 / 10$ & $0 / 4$ & $1 / 14$ & & $0 / 3$ & $0 / 2$ & $0 / 5$ \\
Rectum & $0 / 8$ & $3 / 6$ & $3 / 14$ & & $0 / 3$ & 0 & $0 / 3$ \\
\hline
\end{tabular}

A

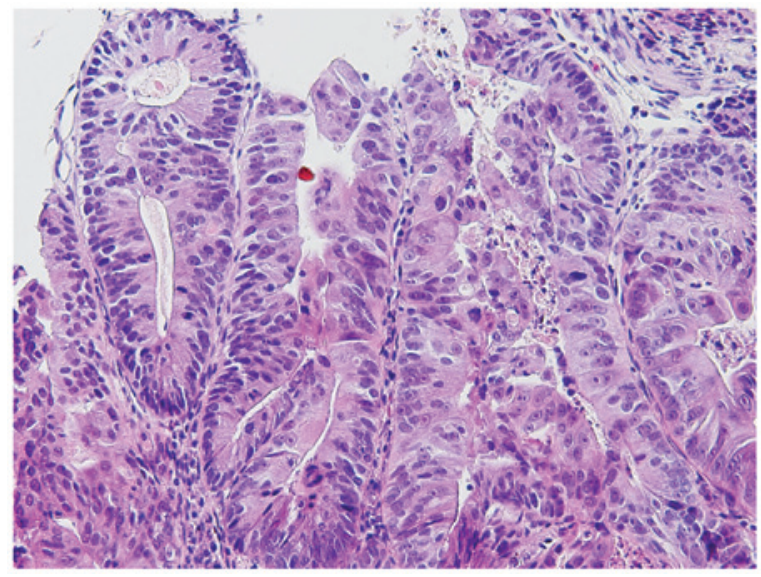

B

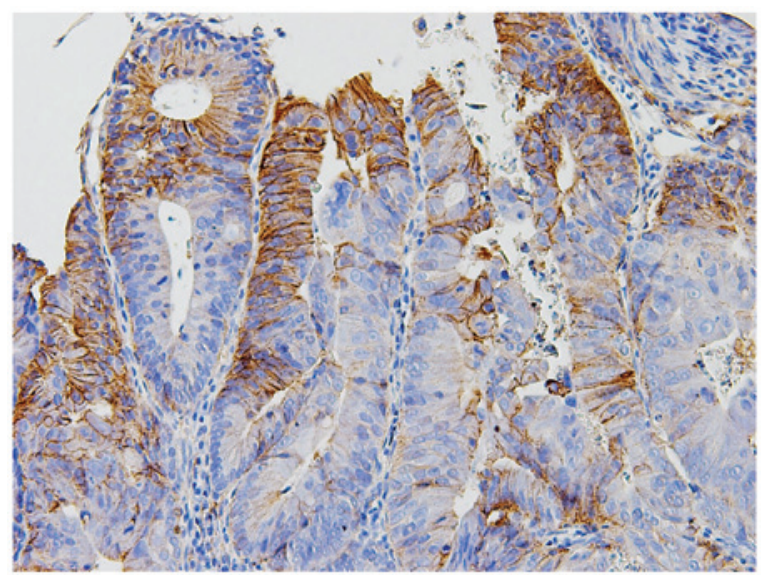

Figure 1. Immunohistochemical staining of EGFR. (A) Hematoxylin and eosin shows a high-power view of moderately differentiated tubular adenocarcinoma of unresectable colorectal carcinoma. (B) Immunohistochemistry shows positive staining for EGFR (31G7) in the cytoplasmic membrane of carcinoma.

and rectum in 17 patients. Right-sided colon carcinomas were frequently identified in elderly patients aged $\geq 65$ years and this tendency was more marked in the female patients (Tables I-III).

Screening of K-ras mutation. When the $\mathrm{pH}$ of buffered formaldehyde is kept neutral, the purified DNA does not degrade. However, our study showed that high molecular weight DNA degrades in unbuffered formaldehyde, as noted in a previous study $(12,15)$. The results of various formaldehyde fixations suggest that DNAse, which plays an important role in degrading genomic DNA during fixation, and other enzymes may degrade genomic DNA. Subsequently, 40 of $49(81.6 \%)$ samples were examined.

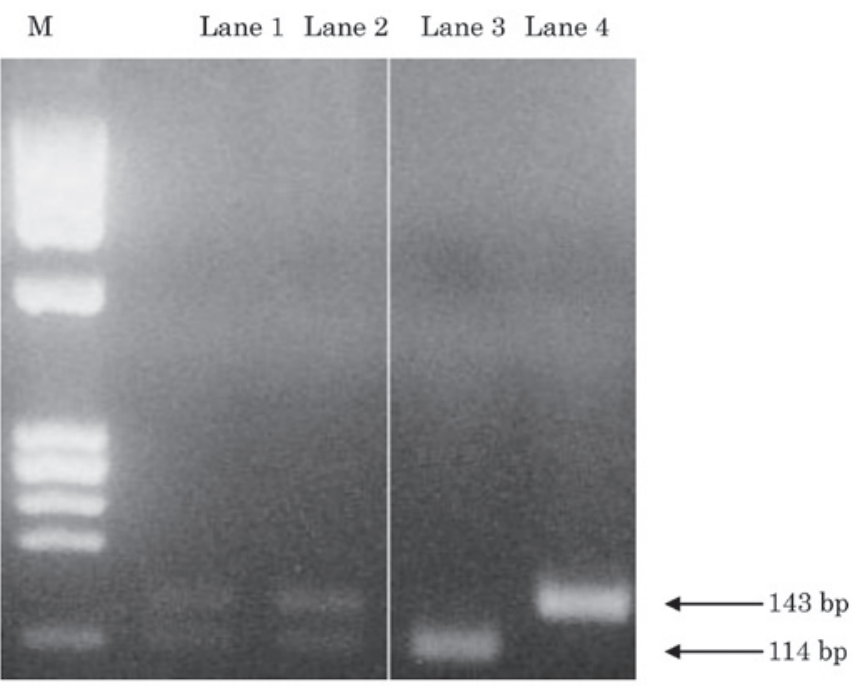

Figure 2. Analysis of $K$-ras codon 12 mutations using polymerase chain reaction-restriction fragment length polymorphism (PCR-RFLP) in unresectable advanced colorectal carcinomas. PCR products were digested with the restriction enzyme $M v a I$. The wild-type and mutant fragments were detected at positions corresponding to 114 and $143 \mathrm{bp}$. Lanes 1 and 2 show a mutant band; lane 3, negative control; lane 4, positive control and M, HaeIII DNA marker.

As shown in Fig. 2, the mutated samples of the $K$-ras codon 12 were screened by PCR-RFLP. The wild-type fragment was digested with a restriction enzyme (MvaI) and detected as a band of $114 \mathrm{bp}$, whereas the mutant-type fragment was not digested and was detected as a band of $143 \mathrm{bp}$. Eight of the $40(20 \%)$ samples had a K-ras codon 12 point mutation (Fig. 2) (Table IV).

No significant correlation was detected between the anatomical subsites, age and $K$-ras mutations, while the incidence of $K$-ras gene mutations was relatively low compared with that noted in previous studies (?).

Result of immunohistochemical staining for EGFR protein. Result of immunohistochemical staining for EGFR protein, carried out with an anti-mouse monoclonal antibody (31G7) on paraffin-embedded tissue sections, was positive in the 40 cases for which screening of $K$-ras mutations was readily detectable.

\section{Discussion}

Colorectal carcinoma has high morbidity and mortality rates worldwide, and according to the estimated number of cancer 
patients identified in 2008 in the GLOBOCAN, published by the International Agency for Research on Cancer (IARC), 1.2 million people had colorectal carcinoma, accounting for approximately $10 \%$ of cancer patients (1). Colorectal carcinoma is the third most common cancer after lung and prostate cancer in males and the second most common cancer after breast cancer in females. Although a high incidence of colorectal carcinoma was not identified in Japan until approximately 1970 , the morbidity rate has rapidly increased since 1975 and it was estimated that $\sim 104,000$ people had colorectal carcinoma in 2005 and $\sim 43,000$ succumbed to the disease in 2008. Over the last 30 years, the incidence of colorectal carcinoma has increased $>5$-fold and the incidence of unresectable advanced/recurrent metastatic colorectal carcinoma (unresectable advanced colorectal carcinomas) has been on the increase with an increase in the incidence of colorectal carcinoma $(2,3)$.

Anti-EGFR antibody (cetuximab), a molecularly targeted agent, was developed as a treatment option for unresectable advanced colorectal carcinomas. In Japan, cetuximab is indicated only for cases of positive EGFR immunostaining and the wild-type ras gene has been investigated for immunohistochemical study. The patients in this study tested positive for EGFR. Standardization of the criteria for EGFR antibody expression and a positive result are necessary, however, a correlation between colorectal carcinoma and EGFR expression was detected in this study.

By contrast, incidence of the mutant $K$-ras gene was lower compared with the controlled statistics in the previously published literature $(15,16)$, likely due to the fact that the wild-type $K$-ras gene colorectal carcinoma is frequently observed in unresectable advanced colorectal carcinomas. The wild-type $K$-ras gene colorectal carcinoma is a candidate lesion of de novo cancer and morphologic type IIc (flat and depressed type). It is known that this type is smaller than the elevated type and that it accelerates the progression of cancer more readily (17). Similar conditions include laterally spreading tumor and non-granular type (LST-NG) (16).

There were 32 males and 17 female patients (mean age, 59.2 years; range, 34-75 years) with unresectable advanced colorectal carcinomas in our hospital. Twenty-four patients were aged $<60$ years with an $\mathrm{M} / \mathrm{F}$ ratio of $16 / 8$ and 25 patients aged $\geq 60$ years with an $M / F$ ratio of $16 / 9$. Additionally, of the patients aged $\geq 65$ years, 15 patients were enrolled as controls and the $M / F$ ratio was $9 / 6$. Concerning the cancer subsite, unresectable advanced colorectal carcinomas developed in the right-sided colon in 13 patients, left-sided colon in 19 patients and rectum in 17 patients. Right-sided colon carcinomas were frequently identified in elderly patients aged $\geq 65$ years, with this tendency being more marked in the female patients.

Furthermore, mucinous carcinoma was observed in two groups including patients in their 30s and elderly patients. Mucinous carcinoma is similar to cancer type associated with abnormalities of germline and somatic line mismatch-repair $(M M R)$ genes in the young and elderly patients, respectively, and the results of the present study together with future studies are likely to further elucidate this type of carcinoma. To confirm that the high incidence of this right-sided colon carcinoma, it is necessary to predict the precursor lesion that develops in the right-sided colon based on the origin of unresectable advanced colorectal carcinomas.
In general, colorectal carcinoma has a multi-step process characterized by a sequence of genetic alterations in cell growth regulatory genes. Mutational activation of the ras gene, in particular the $K$-ras oncogene, is an early event and is considered to play a role in the progression of size and grade of atypia. Ras is part of the ras/raf/MEK/ MAP kinase cascade, which is an essential component of intracellular signaling from activated cell surface receptors to transcription factors in the cell nucleus. Recently, the activating mutation of $b$-raf, a member of the raf gene family, has been found in malignant melanoma and in a wide range of human carcinomas.

Although the incidence of $b$-raf mutation is reported to be $\sim 10 \%$ in colorectal carcinoma $(12,15)$, more recent data $(16)$ have indicated that $b$-raf mutation is associated with a high frequency of microsatellite instability (MSI) and inactivation of the $M M R$ gene in colorectal carcinoma, in particular the so-called serrated neoplasia pathway which may be associated with sessile serrated adenoma/polyp.

Approximately $50 \%$ of the colorectal carcinomas occur in rectosigmoid area, although their relative incidence appears to be on the decrease, in that a shift in location towards the proximal colon during the past few decades has been noted. Noteworthy correlations between certain clinical features and the anatomic subsite of the tumor have been made. Right-sided tumors are said to be more common in the Japanese elderly and in patients with surveillance for clean colon (so-called interval cancer) (18).

As a direct sequence reaction of this study, mutations of $K$-ras codon 13 were detected concurrently with those of codon 12 (data not shown). The mutations of the $K$-ras codon 12 were different transversions: GGT (glycine) to GTT (valine) and GGT (glycine) to TGT (cystein), and the transition: GGT (glycine) to GAT (aspartic acid). In addition, activating mutations involving codon 13 of the $K$-ras gene were detected in three cases. Although the reason for the occurrence of varying mutations remains to be elucidated and data are conflicting, these different mutations seem to be prospectively correlated with the biological behavior or prognosis of the unresectable advanced colorectal carcinomas . To clarify these issues, more studies based on key components (KRAS and BRAF) in the EGFR signal cascade should be carried out.

In conclusion, the increase in the incidence of unresectable advanced colorectal carcinomas in elderly females, located on the right side colon is clinically important and should be elucidated further. Although no investigation on $b$-raf mutations was performed in this study, the abnormality of the $b$-raf gene and the wild-type $K$-ras should be examined for a more accurate analysis of the effect of anti-EGFR antibody (cetuximab).

In addition, the multidisciplinary and multicenter assessments on the anatomic subsites, gender and age in unresectable advanced colorectal carcinomas, suggesting a shift in location towards the right-sided colon in elderly patients is to be demonstrated based on cumulative results, such as the effect of these treatments.

\section{References}

1. Ferlay F, Shin HR and Bray F: Estimates of worldwide burden of cancer in 2008: GLOBOCAN 2008. Int J Cancer 127: 2893-2917, 2010. 
2. Japanese Society for Cancer of the Colon and Rectum: Multi institutional Registry of Large Bowel Cancer in Japan. Case treated in 1999. Vol 28. (Prospective registry date).

3. Watanabe T, Itabashi M, Shimoda Y, et al: Japanese society for cancer of the colon and rectum (JSCCR) guidelines 2010 for the treatment of colorectal cancer. Int J Clin Oncol 17: 1-29, 2012.

4. Ghahremani GG and Dowlatshahi K: Colorectal carcinomas: diagnostic implications of their changing frequency and anatomic distribution. World J Surg 13: 321-325, 1989.

5. Cady B, Stone MD and Wayne J: Continuing trends in the prevalence of right-sided lesions among colorectal carcinomas. Arch Surg 128: 505-509, 1993.

6. Dubrow R, Bernstein J and Holford TR: Age-period-cohort modeling of large-bowel-cancer by anatomic sub-site and sex in Conneticut. Int J Cancer 53: 907-913, 1993.

7. Karapetis CS, Khambate-Ford S, Jonker DJ, et al: K-ras mutations and benefit from cetuximab in advanced colorecta cancer.N Engl J Med 359: 1757-1765, 2008.

8. Vogelstein B, Fearon ER, Hamilton SR, et al: Genetic alterations during colorectal-tumor development. N Engl J Med 319: 525-532, 1988.

9. Snover DC: Update on the serrated pathway to colorectal carcinoma. Hum Pathol 42: 1-10, 2011.

10. Japanese Society for Cancer of the Colon and Rectum (JSCCR) Japanese Classification of Colorectal Carcinoma. Kanehara, Tokyo, pp4-32, 1997.
11. Hamilton SR, Bosman FT, Boffetta P, et al: Carcinoma of the colon and rectum. In: WHO Classification of Tumours of the Digestive System. Bosman FT, Carneiro F, Hruban RH, et al (eds). 4th edition. IARC, Lyon, pp134-146, 2010.

12. Ajiki T, Onoyama H, Yamamoto M, et al: Detection of point mutations in $K$-ras gene at codon 12 in bile from percutaneous transhepatic choledochal drainage tubes for diagnosis of biliary strictures. Int J Pancreatol 18: 215-220, 1995.

13. Lehman TA, Scott F, Seddon M, et al: Detection of $K$-ras oncogene mutations by polymerase chain reaction-based ligase chain reaction. Anal Biochem 239: 153-159, 1996.

14. Jiang W, Kahn SM, Guillem JG, et al: Rapid detection of ras oncogenes in human tumors: applications to colon, esophageal and gastric cancer. Oncogene 4: 923-928, 1989.

15. Fujimori T, Satonaka K, Yamamura-Idei Y, et al: Non-involvement of ras mutations in flat colorectal adenomas and carcinomas. Int J Cancer 57: 51-55, 1994.

16. Kusaka T, Fukui H, Sano Y, et al: Analysis of K-ras codon 12 mutations and p53 overexpression in colorectal nodule-aggregating tumors. J Gastroenterol Hepatol 15: 1151-1157, 2000.

17. Kudo S, Kashida H, Nakajima T, et al: Endoscopic diagnosis and treatment of early colorectal cancer. World J Surg 21: 694-701, 1997.

18. Matsuda T, Fujii T, Sano Y, et al: Five-year incidence of advanced neoplasia after initial colonoscopy in Japan: a multicenter retrospective cohort study. Jpn J Clin Oncol 39: 435-442, 2009. 